\title{
FEASIBILITY INVESTIGATION ON SINGLE PHOTON LIDAR BASED WATER SURFACE MAPPING
}

\author{
G. Mandlburger ${ }^{1,2 *}$, B. Jutzi ${ }^{3}$ \\ ${ }^{1}$ University of Stuttgart, Institute for Photogrammetry, Germany - gottfried.mandlburger@ifp.uni-stuttgart.de \\ ${ }^{2}$ TU Wien, Department of Geodesy and Geoinformation, Austria - gottfried.mandlburger@ geo.tuwien.ac.at \\ ${ }^{3}$ Karlsruhe Institute of Technology (KIT), Institute of Photogrammetry and Remote Sensing, Germany - boris.jutzi@kit.edu
}

Commission I, WG I/2

KEY WORDS: Laser Bathymetry, Water Level Estimation, Single Photon LiDAR, Multi-Photon LiDAR, Discrete Echo LiDAR

\begin{abstract}
:
The recent advent of single photon sensitive airborne LiDAR (Light Detection And Ranging) sensors has enabled higher areal coverage performance at the price of an increased outlier rate and a lower ranging accuracy compared to conventional Multi-Photon LiDAR. Single Photon LiDAR, in particular, uses green laser light capable of penetrating clear shallow water. Although primarily designed for large area topographic mapping, the technique can also be used for mapping the water surface and shallow water bathymetry. In this contribution we investigate the capability of Single Photon LiDAR for large area mapping of water surface heights. While interface returns from conventional green-only bathymetric sensors generally suffer from water level underestimation due to the water penetration capabilities of green laser radiation, the specific questions are, if Single Photon LiDAR (i) is less affected by this well known effect due to the high receiver sensitivity and (ii) consequently delivers a higher number of water surface echoes. The topic is addressed empirically in a case study by comparing the water surface responses of Single Photon LiDAR (Navarra, Spain) and Multi-Photon Topo-Bathymetric LiDAR (Neubacher Au, Austria) for selected water bodies with a horizontal water surface (reservoirs, ponds). Although flown at different altitudes, both datasets are well comparable as they exhibit the same strip point density of ca. 14 points $/ \mathrm{m}^{2}$. The expected superiority of Single Photon LiDAR over conventional green-only bathymetric LiDAR for mapping water surfaces could not be verified in this investigation. While both datasets show good agreement compared to a reference water level when aggregating points into cells of $10 \times 10 \mathrm{~m}^{2}$ (mean deviations $<5 \mathrm{~cm}$ ), higher resolution Single Photon LiDAR based water surface models (grid size $1-5 \mathrm{~m}$ ) show a systematic water level underestimation of 5-20 cm. However, independently measured ground truth observations and simultaneous data acquisition of the same area with both techniques are necessary to verify the results.
\end{abstract}

\section{INTRODUCTION}

High resolution, area wide mapping of water level heights is important for many fields of application like hydrology, hydraulic engineering, and ecology, but capturing water surfaces is challenging for active or passive remote sensing (Thomas and Guenther, 1990; Rupnik et al., 2015; Mandlburger et al., 2013). In hydro sciences, water surface information is required for discharge estimation and for the calibration of multi-dimensional hydrodynamic-numerical models. Whereas the demand regarding spatial resolution is moderate for these applications, precise knowledge of the elevation and attitude of the air-water interface is required in bathymetric LiDAR (Light Detection And Ranging) for every laser pulse to correct the raw signal due to beam refraction at the interface layer (Guenther et al., 2000).

The recent advent of LiDAR sensors using low energy pulses and detectors that are sensitive to individual photons has increased the areal coverage performance compared to conventional LiDAR sensors (Stoker et al., 2016) at the price of a reduced ranging accuracy and a higher measurement noise (Ullrich and Pfennigbauer, 2016). Especially Single Photon LiDAR, originally developed by Sigma Space Corporation and now available as a Leica/Hexagon product (SPL100), is suitable for both topographic and bathymetric mapping (Degnan, 2016).

In this contribution we investigate the capability of Single Photon

${ }^{*}$ Corresponding author
LiDAR operating with very short laser pulses (400 ps) in the visible, green domain of the spectrum $(\lambda=532 \mathrm{~nm})$ for large area water surface mapping. The research questions are, (i) if the single photon sensitivity enables a high density of water surface echoes and (ii) if water surface level underestimation is less compared to conventional LiDAR. The underestimation effect is well known from literature for green laser radiation (Thomas and Guenther, 1990; Zhao et al., 2017; Mandlburger et al., 2013). We address the topic empirically by comparing Single Photon and MultiPhoton LiDAR point clouds of selected horizontal water bodies (reservoirs, ponds).

The remainder of this article is structured as follows. In Section 2 the basics of Single Photon LiDAR are briefly summarized and compared to conventional Multi-Photon technology. The study areas and processing steps are detailed in Section 3 and the results of the case study are presented and critically discussed in Section 4. Finally, the main findings are summarized in Section 5.

\section{SINGLE PHOTON AND CONVENTIONAL LIDAR TECHNOLOGY}

If a new sensor technology like Single Photon LiDAR arises, the terminology is often vague and confusing due to the different wording of the developers, researchers, and customers. The evaluation of the new sensor technology compared to established technology is arduous because the techniques themselves are often specific and sometimes not comparable at all. Therefore, 
in the following the principles of Single Photon LiDAR (Leica SPL100) are described and compared to established conventional Multi-Photon LiDAR in general and to a specific TopoBathymetric LIDAR system (RIEGL VQ-880-G) in particular. The specifications are derived from company brochures (Leica, 2017; Riegl, 2017), internet research, or literature (Degnan, 2016; Stoker et al., 2016; Jutzi, 2017).

The Single Photon LiDAR technology is characterized by two essential optical components: (i) a Diffractive Optical Element (DOE) splitting the laser beam into an array of $10 \times 10$ beamlets and (ii) a Optical Receiver consisting of photosensitive subarrays, where each sub-array is aligned to a beamlet and contains numerous photosensitive detector elements. These optical components ensure an efficient illumination of the photosensitive sub-array and additionally avoid optical crosstalk.

i By the Diffractive Optical Element an array of $10 \times 10$ beamlets is provided. For a single beamlet the given beam divergence of $0.08 \mathrm{mrad}$ is supposed to approximately merge the entire instantaneous FoV (iFoV) of the relevant photosensitive sub-array with numerous detector elements (sometimes referred to as micro-cells), but unfortunately the $\mathrm{iFoV}$ is not specified by the manufacturer.

ii The Optical Receiver consists of numerous photosensitive detector elements, but again it is not officially specified by the companies which kind of detector is utilized. For multiple single photon detection, inexpensive Micro Channel Plate PhotoMultiplier Tubes (MCP-PMT) or Silicon PhotoMultipliers (SiPM), or Single Photon Avalanche Diode (SPAD) arrays are more or less suitable. The very low jitter time of the detector element is sometimes specified by the companies with $50-100 \mathrm{ps}$ (is equivalent to $0.75-1.5 \mathrm{~cm}$ in range), which has a significant impact on the ranging accuracy. Furthermore, a low recovery time (1.6 ns equivalent to $24 \mathrm{~cm}$ in range) is important for daytime operation as reflections from the laser or sun light at particles in the atmosphere may trigger detection events. The low recovery time essentially enables multi-target capabilities and allows promising vegetation and bathymetry measurements.

The Single Photon LiDAR instrument SPL100 from Le$\mathrm{ica} /$ Hexagon operates with a wavelength of $532 \mathrm{~nm}$. While this visible green wavelength generally exhibits low reflectance values for natural surfaces (soil/dry vegetation $15 \%$ and green vegetation $10 \%$ ), it is advantageous for LiDAR because the optical components are inexpensive, the detector elements show a high efficiency, detector dark count contributions to background noise are typically much lower, and the good transmission characteristic in water supports topographic as well as bathymetric mapping with a single instrument (Degnan, 2016).

Furthermore the instrument design is optimized to gain a maximum point density. The Single Photon LiDAR is tuned for Photon Detection Probability (PDE) of 0.95 for a $10 \%$ surface reflectance (e.g. green vegetation) ${ }^{*}$ and a PDE of 0.99 for a $15 \%$ surface reflectance (e.g. soil/dry vegetation). With a maximum flying height of $4500 \mathrm{~m}$ above ground level (AGL), the derived areal coverage and the corresponding point density of Single

${ }^{*}$ We use the term PDE in this paper as it commonly used in subject literature, but we note that it rather represents an object detection probability in this context.
Photon LiDAR is much higher compared to conventional MultiPhoton LiDAR.

In the aquatic domain, the water surface itself, the submerged water bottom, and the dry ground surface in the littoral area are challenging to measure with LiDAR in a single campaign. Considering the short laser pulse width $(400 \mathrm{ps}$, equivalent to $6 \mathrm{~cm}$ in range) and the low jitter time $(0.75 \mathrm{~ns}$, equivalent to $1.5 \mathrm{~cm}$ in range) of Single Photon LiDAR, an accurate surface ranging can be expected for the water level as well as for the (shallow) water bottom with the first photon detection. In comparison, the MultiPhoton LiDAR laser pulse width (1-5 $\mathrm{ns}$ is equivalent to $15-75 \mathrm{~cm}$ in range) is much larger and waveform processing is challenging (Jutzi and Stilla, 2003, 2005) especially due to the different speed of light in the medium air and water. Even if the first photon detection for Single Photon LiDAR is not caused by the water surface it is very likely that a measurement is derived from the water column below the water surface or the water bottom. For second photon detection, the low recovery time of Single Photon LiDAR ( $1.6 \mathrm{~ns}$ is equivalent to $24 \mathrm{~cm}$ in range) is relevant.

In this contribution, SPL100 point clouds are compared to MultiPhoton LiDAR point clouds captured with the Topo-Bathymetric VQ-880-G sensor. The latter records the full-waveform profile of each backscattered laser pulse with $2 \mathrm{GHz}$ enabling sophisticated offline waveform analysis in post-processing, but also performs online waveform processing (Pfennigbauer and Ullrich, 2010). The received waveform is hereby processed in real-time and the shape of the waveform in the vicinity of a local maximum is analyzed. Next to the raw amplitude $A$ and range $R$, the following quantities are provided for each echo: (i) calibrated amplitude (i.e. amplitude measure $[\mathrm{dB}]$ proportional to the instruments' detection limit), (ii) relative reflectance (i.e. difference $[\mathrm{dB}]$ between the measured amplitude and the theoretical amplitude of a diffusely reflecting object with known reflectivity at a distance of $R$ ), and (iii) pulse shape deviation (i.e. a measure describing the deviation of the measured echo pulse from an ideal single object return with orthogonal incidence of the laser beam). It is noted here that although both Single Photon LiDAR and MultiPhoton LiDAR in general and the respective instruments used in this investigation (SPL100, VQ-880-G) in particular provide signal strength information, the investigation at hand entirely focuses on the geometry of the obtained discrete echo point clouds.

\section{STUDY AREAS AND APPLIED EVALUATION METHOD}

In this section the study areas are introduced (Section 3.1) and the applied evaluation method is presented (Section 3.2).

\subsection{Study Area}

In 2017 the government of Navarra (Spain) commissioned a flight campaign for capturing the entire province area with a Leica SPL100 sensor. From the area-wide dataset, the company Trascasa provided the unfiltered point cloud of six areas featuring different inland water bodies (rivers, reservoirs; cf. Figure 1). As no external water level reference data were available, four reservoirs with horizontal water level were chosen as the specific study areas (A01, A03-05, cf. Figure 1).

The 3D point clouds were captured from a flying altitude of about $4200 \mathrm{~m}$ AGL resulting in a single flight line swath width of $2260 \mathrm{~m}$ for the employed conical scanning (Palmer scanner) with 
Table 1. Specifications for Single Photon LiDAR, Multi-Photon LiDAR, and Topo-Bathymetric LiDAR. For generalization purposes, a standard combination of specifications from various companies is provided for Multi-Photon LiDAR.

\begin{tabular}{|c|c|c|c|}
\hline & Single Photon LiDAR & Multi-Photon LiDAR & Topo-Bathymetric LiDAR \\
\hline Type & Leica/Hexagon SPL100 & various & RIEGL VQ-880-G \\
\hline Laser wavelength & $532 \mathrm{~nm}$ & $532 / 1064 / 1550 \mathrm{~nm}$ & $532 \mathrm{~nm}$ \\
\hline Laser pulse width (FWHM) & $400 \mathrm{ps}$ & $1-5 \mathrm{~ns}$ & $1.6 \mathrm{~ns}$ \\
\hline Laser beam divergence @ 1/e & $\sim 0.08 \mathrm{mrad} / \mathrm{beamlet}$ & $0.25-1 \mathrm{mrad}$ & $0.7-1 \mathrm{mrad}$ \\
\hline Field-of-View (FoV) & $20,30,40$ or $60^{\circ}$ & $\leq 72^{\circ}$ & $40^{\circ}$ \\
\hline Detector elements & numerous & 1-2 PIN/APD & 1-2 APD \\
\hline Intensity measurement & available & available, up to 16 bit & available, up to 16 bit \\
\hline Minimum surface detection & 1 photon & $250-1000$ photons & 250 photons \\
\hline Instantaneous FoV (iFoV) & N/A & $0.25-1 \mathrm{mrad}$ & $0.7-1 \mathrm{mrad}$ \\
\hline Jitter timing / ranging precision & $50-100 \mathrm{ps} / 0.75-1.5 \mathrm{~cm}$ & $50-500 \mathrm{ps} / 0.75-7.5 \mathrm{~cm}$ & N/A / $2.5 \mathrm{~cm}$ \\
\hline Dead time/recovery time & $1.6 \mathrm{~ns}$ & N/A & N/A \\
\hline Pulse repetition rate (PRR) & $60 \mathrm{kHz}$ & $\leq 1000 \mathrm{kHz}$ & $550 \mathrm{kHz}$ \\
\hline Max. flying altitude (AGL) & $\leq 4500 \mathrm{~m}$ & $\leq 5000 \mathrm{~m}$ & 2500-3600/600 m (Topo/Bathy) \\
\hline Areal coverage @ 8 pts $/ \mathrm{m}^{2}$ & $\leq 2000 \mathrm{~km}^{2} / \mathrm{h}$ & $\leq 450 \mathrm{~km}^{2} / \mathrm{h}$ & $\leq 450 \mathrm{~km}^{2} / \mathrm{h}$ (Topo) \\
\hline
\end{tabular}

a constant off-nadir angle of $15^{\circ}$. At a flying speed of 180 knots, the effective scan rate of $6 \mathrm{Mhz}$ (PRR: $60 \mathrm{kHz}, 100$ beamlets per laser pulse) results in an average (last echo) point density on dry ground of 14.5 points $/ \mathrm{m}^{2}$ with a considerably larger point density on the strip boundary due to the circular (Palmer) scan pattern. The sensor stores the 3D coordinates $(\mathrm{x}, \mathrm{y}, \mathrm{z})$ and time stamps (t) of discrete echoes with additional attributes (signal strength, RGBI, scan angle, etc.). The signal strength hereby relates to the number of nearly synchronously triggered micro-cell array elements in response to the laser beamlet hitting an (and reflecting from an) object within the beamlets' iFoV. The color information is derived from the incorporated RCD30 80 MPix RGBI camera. All mission parameters are additionally listed in Table 2.

Whereas this contribution mainly focuses on the properties of Single Photon LiDAR point clouds reflected from water bodies, a conventional Topo-Bathymetric dataset captured with a RIEGL VQ-880-G sensor at the Pielach River (Austria) is used as comparison basis. A detailed description of the investigation area, a natural conservation located in the eastern part of Austria (cf. Figure 2, can be found in Mandlburger et al. (2015). The captured area features more than a dozen groundwater ponds, each of which exhibiting a constant water level. Data acquisition took place in November 2017 from a flying altitude of $650 \mathrm{~m}$ AGL with a flying velocity of 110 knots. Similar to the Single Photon LiDAR system, the scan pattern is circular, but the constant off-nadir angle is $20^{\circ}$ resulting in swath width of $480 \mathrm{~m}$. For this single-receiver instrument, the PRR equals the effective scan rate of $550 \mathrm{kHz}$, which yields a point density over land surfaces of 14 points $/ \mathrm{m}^{2}$, closely matching the the Single Photon LiDAR point density. Discrete laser echoes (3D position, amplitude, reflectance, echo pulse shape deviation) are obtained from online waveform processing (Pfennigbauer and Ullrich, 2010). While the waveforms are additionally stored for off-line postprocessing, this is not employed in this investigation, but the comparison is carried out on the discrete echo point clouds only.

It is stated here that an off-nadir angle of $15-20^{\circ}$ is considered the optimum for bathymetric applications (Guenther et al., 2000), thus both systems are well suited for the investigation at hand.

\subsection{Evaluation Method}

For evaluating the feasibility of Single Photon LiDAR 3D point clouds for area wide water surface mapping, first a couple of preprocessing steps were carried out (quality checks, point cloud filtering and classification), then a reference water surface level

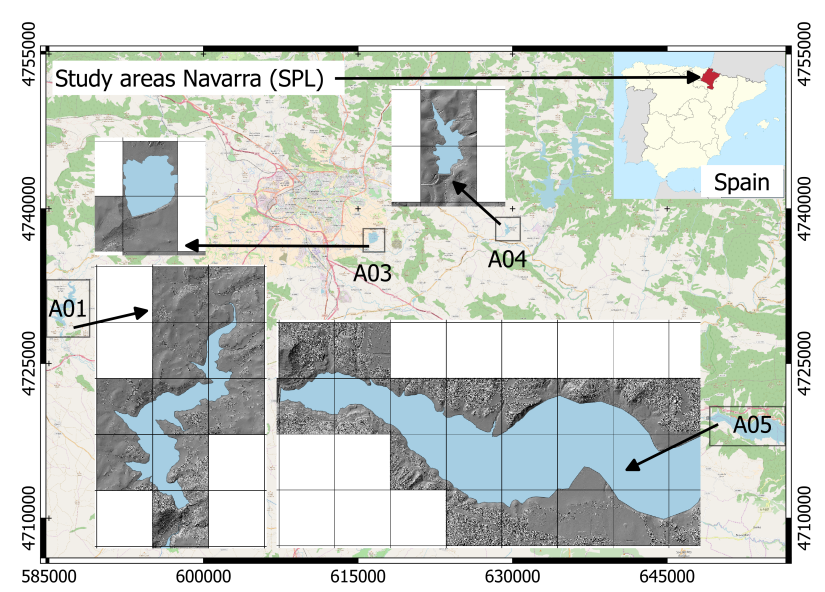

Figure 1. Single Photon LiDAR area Navarra; City of Pamplona area (OpenStreetMap) with selected reservoirs (A01, A03-05; gray boxes), coordinate frame: WGS84/UTM 30N; Details: terrain relief map of reservoir areas, coordinate grids: $1 \mathrm{~km}$.

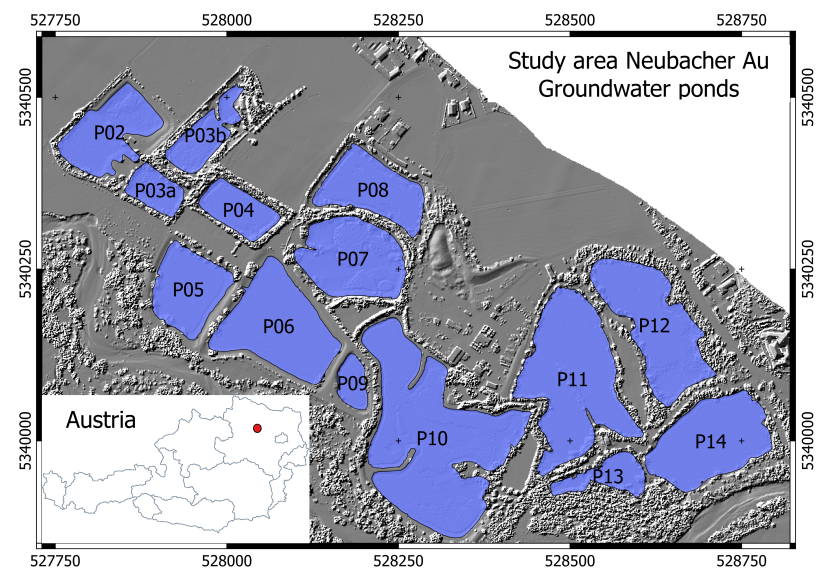

Figure 2. Topo-Bathymetric LiDAR area Neubacher Au, Pielach River, Austria with depicted ground water ponds (blue polygons), coordinate frame: ETRS89/UTM 33N.

as well as various water surface raster models were determined for each reservoir and pond based on all points classified as water (water surface, water column, water bottom), and finally the results were evaluated. The following processing steps were applied to both Single Photon and Multi-Photon LiDAR datasets. 


\begin{tabular}{|l|l|r|r|}
\hline & unit & SPL & MPL \\
\hline Flying velocity & {$[\mathrm{knots}]$} & 180 & 110 \\
\hline Flying altitude & {$[\mathrm{m}]$} & 4200 & 650 \\
\hline Effective scan rate & {$[\mathrm{kHz}]$} & 6000 & 550 \\
\hline Strip swath width & {$[\mathrm{cm}]$} & 2260 & 480 \\
\hline Footprint diameter & {$[\mathrm{cm}]$} & 35 & 70 \\
\hline Precision/roughness & {$[\mathrm{cm}]$} & 3.0 & 1.3 \\
\hline Strip fitting precision & {$[\mathrm{cm}]$} & 3.9 & 1.2 \\
\hline Point density (land/water) & {$[\mathrm{cm}]$} & $14.5 / 7.0$ & $14.0 / 12.5$ \\
\hline Total FOV & {$\left[{ }^{\circ}\right]$} & 30 & 40 \\
\hline
\end{tabular}

Table 2. Flight mission parameters and selected quality control measures for Single Photon LiDAR (SPL, Navarra) and Multi-Photon LiDAR (MPL, Neubacher Au).

Data preprocessing started with a volumetric density analysis in order to eliminate clutter points above and below the terrain stemming from occasional reflections of the laser pulses at particles in the atmosphere. Such points are typical for Single Photon LiDAR due to the single photon sensitivity of the receiver. The same applies, albeit to a lesser extent, to high-sensitive MultiPhoton LiDAR sensors used for Topo-Bathymetric applications (cf. Figure 3).

To assess (i) the general noise level of the point clouds and the derived DEMs thereof, (ii) the strip fitting precision, and (iii) the achieved point densities, a standard ALS quality control procedure implemented in the scientific laser scanning software OPALS (Pfeifer et al., 2014) was subsequently carried out using the last echo points. The noise level and the strip fitting precision was hereby assessed via analysis of $0.5 \mathrm{~m}$-DEM raster models interpolated with a moving least squares approach. For each grid post a best fitting plane is estimated from the surrounding $\mathrm{k}$ nearest neighbors $(\mathrm{k}=15)$ and the resulting $\sigma_{0}$ can be interpreted as a roughness measure. At smooth surfaces (sealed roads, house roofs, etc.) $\sigma_{0}$ characterizes the inherent precision of the point clouds. As reported in Table 2, Multi-Photon expectedly outperformed Single Photon LiDAR w.r.t. smoothness $(3.0 \mathrm{~cm}$ vs. $1.3 \mathrm{~cm})$ and strip fitting precision $(3.9 \mathrm{~cm}$ vs. $1.2 \mathrm{~cm})$ due to the shorter ranges and the higher single point reliability being the result of relying on approximately 250 photons for a single range measurement instead of only a few photons in the Single Photon LiDAR case. Apart from that, the overall quality is high for both datasets with strip-to-strip deviations way below $10 \mathrm{~cm}$.

For deriving a reference water level for each water body (Single Photon LiDAR: reservoirs, Multi-Photon LiDAR: ponds), the following two strategies are proposed: Considering the very strong absorption of infrared (IR) radiation at water, the IR information available for the Single Photon LiDAR point clouds via mapping the colors from the RCD30 images can be used to delineate the shoreline, referred to as water-land-boundary (WLB) in the following. By interpolating an IR-raster, the WLB is found by deriving the contour line at the appropriate IR-level characterizing the water-land-transition. Interpolating the heights along this line from the point cloud finally results in a good estimate of the horizontal water level of a standing water body. While this would have been the preferred approach as it allows a fully automated processing chain, it could not be applied to the data at hand due to geometric and radiometric artifacts. The available point colors showed pronounced block effects with the color of water changing from black (as expected for IR) to $75 \%$ gray from one processing unit to the next. Furthermore, geometric displacements at block boundaries of up the $2.5 \mathrm{~m}$ were detected and prevented the application of the IR-channel based reference water level estimation.
As an alternative, a first approximate water level was detected interactively by analyzing the $3 \mathrm{D}$ point cloud in vertical sections in a $3 \mathrm{D}$ editor. As green radiation tends to penetrate into the topmost level of the water column, the approximate water level should rather be defined slightly above the actual water level. This rough estimate allows a first classification of dry and submerged points. The precise water level is subsequently derived as the $99.5 \%$ quantile computed from the elevation histogram of all submerged points. This way, occasional points above the water surface are filtered out. Lacking independently measured ground truth, the water level derived in such a way serves as the basis for the subsequent evaluation of both Single Photon and MultiPhoton LIDAR-derived higher resolution water surface models.

For the derivation of Digital Water Surface models (DWSM) in regular grid structure with $1 \mathrm{~m}, 2 \mathrm{~m}, 5 \mathrm{~m}$, and $10 \mathrm{~m}$ grid spacing, we follow the statistical approach of Mandlburger et al. (2013). In each cell, the approach accumulates all submerged points with a maximum water depth of $50 \mathrm{~cm}$ and calculates the elevation histogram. Different models, each using a specific quantile (90\%, $95 \%, 98 \%, 99 \%, 100 \%$ ), are calculated and evaluated against the reference water level by computing Digital Elevation Models of Differences (DoD) storing the height difference between the reference level and the estimated cell level. While it is clear that this rather simple approach is only applicable to water bodies with horizontal water surface, it still allows addressing the tackled research question of the feasibility of Single Photon LiDAR for water surface mapping when selecting appropriate water bodies. This is definitely the case for the chosen dammed reservoirs (Single Photon LiDAR, Navarra) and the ground water ponds (MultiPhoton LiDAR/Topo-Bathymetric, Neubacher Au).

\section{RESULTS AND DISCUSSIONS}

In this section, the results of data processing are presented (4.1 and 4.2) and critically discussed considering the specific properties of Single Photon and Multi-Photon LiDAR (4.3).

\subsection{Data Preprocessing}

The first step within the data processing pipeline was the density based clutter point detection and a raw delineation of dry and wetted areas. Figure 3 show perspective views of exemplary Single Photon LiDAR (Navarra) and Topo-Bathy/Multi-Photon LiDAR (Neubacher $\mathrm{Au}$ ) point clouds. It can be seen that the Single Photon LiDAR dataset exhibits a considerable number of clutter points above the surface. As the system detects echoes for each beamlet as soon as a few photons arrive at the detector array, reflections from aerosol particles can spontaneously trigger echo detection. The volumetric density of these clutter points, however, is low compared to the density if the laser beam hits objects on the earths' surface. Points were classified as outliers, if less than 3 points were found within a $50 \mathrm{~cm}$-sphere (cf. green points in Figure 3). Although the Topo-Bathymetric Multi-Photon LiDAR sensor (cf. Figure 3, right) is also tuned for detecting weak signals from the water body, the dataset only contains a few clutter points as around 250 photons are necessary to trigger a target event. Furthermore, Figure 3 shows the water points (blue) and the bare earth/vegetation points (red) in different colors as a result of the interactively estimated approximate water level.

Data preprocessing furthermore contained a full quality check of the strip-wise 3D point clouds including last-echo point density estimation. While the quantitative density estimates are reported 


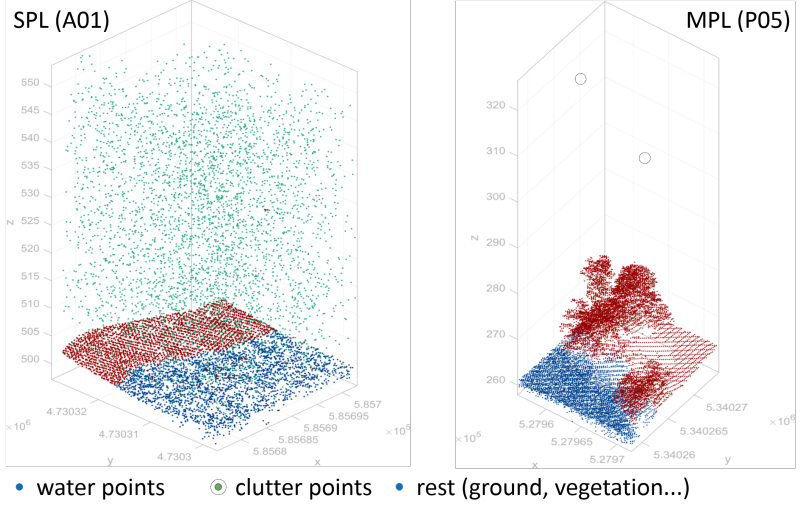

Figure 3. Perspective views of 3D point clouds; Single Photon LiDAR (left) and Multi-Photon LiDAR (right).

in Table 2 separately for water and dry land, Figure 4 shows the locally varying point densities as color coded maps. For the Single Photon LiDAR dataset, test area A01 shows a noticeable point density drop in water of ca. $50 \%$ compared to the surrounding land (land: 14.5 points $/ \mathrm{m}^{2}$, water: 7 points $/ \mathrm{m}^{2}$, cf. Table 2), which is representative for all studied Single Photon LiDAR areas. The average water point density drop is less for the MultiPhoton LIDAR dataset as can be seen from both the right side of Figure 4 and Table 2 (land: 14 points $/ \mathrm{m}^{2}$, water: 12.5 points $/ \mathrm{m}^{2}$ ). However, a considerable density variation can be observed for the individual ponds ranging from almost no (e.g. P04-06) to a pronounced (P07, P10) density drop. This aspect is analyzed and discussed in more detail below. For both Single Photon and MultiPhoton LIDAR, a much higher point density can be observed at the strip boundary due to the circular scan pattern. While the extreme strip boundary point density $\left(>50\right.$ points $\left./ \mathrm{m}^{2}\right)$ is unnecessary for capturing topography, it is well advantageous for capturing water surfaces as will be shown later in this section.

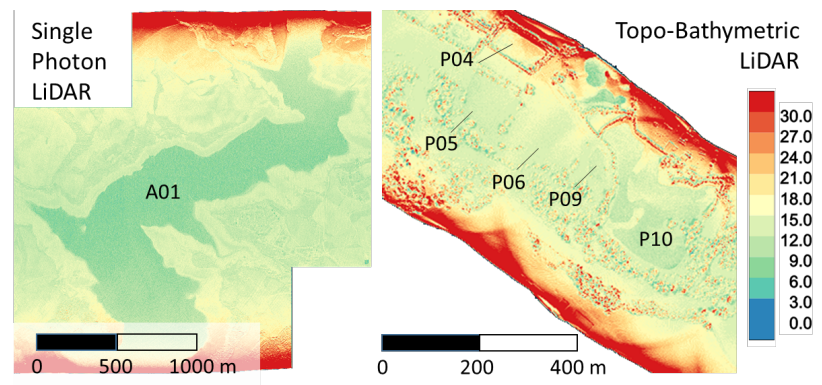

Figure 4. Color coded point density maps for Single Photon (left) and Multi-Photon LiDAR (right).

\subsection{Water Level and Qualitative Assessment}

As lined out in Section 3.2, a representative water level was estimated for each of the studied water bodies using a histogram based, statistical analysis of the water point elevations $(99.5 \%$ quantile). While this simple strategy is regarded suitable for the selected reservoirs and ponds featuring a constant water level over the entire domain, it is clear that small local water level fluctuations due to wind waves cannot be modeled with this approach. As the primary focus of the paper is to investigate if the single photon sensitivity results in a more concise response of the green laser signal from the water surface, the main emphasis is lain on a qualitative and quantitative assessment of the water level underestimation. The prior is addressed by visual comparison of selected near-shoreline point cloud transects (cf. Figure 5) and the latter by evaluating the height differences of the estimated reference water level with the spatially varying height estimates obtained from different water surface raster models (cf. Section 4.3).

Figure 5 shows point cloud transects in the littoral area of selected Single Photon (left) and Multi-Photon LiDAR (right) datasets. Each plot contains the calculated reference water level as a red horizontal line and the classified points within a $3 \mathrm{~m}$ buffer (blue: water candidate points, orange: land/vegetation). The displayed transects reveal variations in overall point density and water level underestimation. For the Single Photon LiDAR area A01, two transects from the border (a) and the center (b) of the strip are displayed. Whereas the higher point density on the strip border increases the likelihood for near water surface laser echoes, in general most of the laser echoes penetrate more or less into the topmost layer of the water column. Although unexpected, this is an important finding as it means that the single photon sensitivity does not necessarily lead to a better water level estimation. The smaller laser footprint (Single Photon LiDAR: $35 \mathrm{~cm}$, MultiPhoton LiDAR: $70 \mathrm{~cm}$ ) may hereby have a negative impact on the water surface detection probability for direct (specular) reflections from the interface. While it is clear from physics of (green) light interaction with water in general and the laser radar equation (Tulldahl and Steinvall, 2004) in particular that most of the direct interface reflections are not back-scattered within the receivers' FOV due to specular reflection for a $15^{\circ}$ off-nadir laser beam, it is still remarkable that volume back-scattering in the first centimeters of the water column is often not sufficient to immediately trigger an echo. However, this statement only holds for the flight mission parameters chosen for the campaign at hand, optimized for capturing large area topography.

Figure 5c, taken from the Single Photon LiDAR area A04, presents a case with an extremely low overall water point density. While water surface estimation is hardly possible for this clear water reservoir, mapping the submerged shallow water bottom surface becomes feasible. It should be stated here that the SPL100 is mainly designed for topographic mapping, but the green laser wavelength $(\lambda=532 \mathrm{~nm})$ inherently provides bathymetric capabilities. The shown example indicates a direct relationship between turbidity and discrete echo point density. But reliable statements in this respect, especially in the context of Single Photon LiDAR, would need in-depth investigations and are not further discussed here.

In contrast to the Single Photon LiDAR examples, the TopoBathymetric Multi-Photon LiDAR datasets generally show a higher number of laser echoes close to the reference water surface. This especially applies to the examples displayed in Figure $5 \mathrm{~d}$ and e (P02, P05). As in both cases laser echoes showing only minimal sub-surface penetration can be found within a radius of ca. $1 \mathrm{~m}$, water surface reconstruction with high spatial resolution is feasible. This property, however, is required to allow water surface mapping for tilted surfaces like running or wavy water water bodies, where spatial aggregation would lead to accuracy losses due to over-smoothing.

Figure 5d-f furthermore exhibit a vertical gap between points from the submerged bottom and the water column. This is a specific property of Multi-Photon LiDAR using larger pulse lengths compared to Single Photon LiDAR. In general, the range discrimination of every LiDAR system (i.e. the minimum distance 
between two consecutive objects of a multi-return LiDAR system) is limited by the pulse length and the receiver band width. For the RIEGL VQ-880-G sensor using object detection based on the recorded echo waveform, the range discrimination distance is limited by the laser pulse length (ca. $1.6 \mathrm{~ns}=24 \mathrm{~cm}$ ) which corresponds well to the size of the data gap above the submerged bottom in Figure 5d-f. Specifically in the littoral zone, the higher reflectance of the shallowly submerged bottom shields echoes from the water surface, exemplified in Figure 5e. It is further noted here that this limitation also applies to the SPL100, as the systems' recovery time is in the same range as the VQ- $880-\mathrm{G}$ pulse length (1.6 ns). However, due to the higher Single Photon LiDAR point density and the characteristic of immediately reacting to incoming photons, the above mentioned gap between bottom and water column points is not noticeable in Figures 5a-c.

The above mentioned relationship between water column point density and turbidity also becomes apparent for the TopoBathymetric Multi-Photon LiDAR datasets. Whereas P02 (Figure $5 \mathrm{~d}$ ) shows a rather homogeneous volumetric point density over the entire displayed $2 \mathrm{~m}$ depth range, P05 (Figure 5e) shows a decreasing density with increasing depth. For P11 (Figure 5f), the echoes are condensed around the water surface with a rapid point density drop both in the water column and at the bottom surface. This corresponds to the in-situ measured Secchi depths (P02: $3.50 \mathrm{~m}, \mathrm{P} 05: 1.95 \mathrm{~m}, \mathrm{P} 11: 0.80 \mathrm{~m}$ ), but further analysis and reference data would be necessary to verify this statement.

\subsection{Quantitative Assessment}

To assess the feasibility of Single Photon LiDAR for highresolution water surface mapping quantitatively, multiple water surface raster models with cell sizes of $1 \mathrm{~m}, 2 \mathrm{~m}, 5 \mathrm{~m}$, and $10 \mathrm{~m}$ have been calculated for each test area as described in Section 3.2. For the $2 \mathrm{~m}$ rasters, the deviations from the reference water level are depicted in Figure 6 for investigation areas A01, A03, and A05 (Single Photon LiDAR) and P02, P06, and P12 (TopoBathymetric Multi-Photon LiDAR), respectively. It can clearly be seen that the water level underestimation is generally less for the Multi-Photon datasets as indicated by the whitish and yellowish color tones $(0-8 \mathrm{~cm})$ compared to the dominant red colors for the Single Photon datasets $(8-20 \mathrm{~cm})$. The reflectance of the water surface and the topmost layer of the water column is, thus, not sufficient to trigger water surface echoes reliably even though the SPL100 features a detection probability of $95 \%$ for objects with $10 \%$ reflectivity. Again, as already stated in Section 4.2, a relation with turbidity is apparent, as water level underestimation is less for area A01 compared to A03 and A05. The occasional gray dots in $\mathrm{A} 03$ and $\mathrm{A} 05$ indicate data voids, i.e. no water point was found in a $2 \times 2 \mathrm{~m}^{2}$ cell within a $50 \mathrm{~cm}$ band below the water surface.

Although Multi-Photon LiDAR performs better in this respect, accurate water level results can still not be achieved for all tested areas (cf. P12 in Figure 6). In general, the findings in this investigation are in line with Mandlburger et al. (2013), who concluded that water surface estimation based on discrete green-only $3 \mathrm{D}$ points requires (i) appropriate spatial aggregation and the use of higher quantiles (e.g. $99 \%$ for the histogram based water level height estimation within each cell. The required aggregation level hereby depends on the overall water point density, which is correlated with turbidity and water surface roughness. To complete the picture, Table 3 lists the mean deviations from the reference water level for selected test areas based on the $99 \%$ quantile and different aggregation levels.
It can be read from the last column of Table 3 that water levels can be derived from both Single Photon and Multi-Photon 3D point clouds with a mean absolute deviation of less then $0.05 \mathrm{~m}$ for all tested water bodies when aggregating the points in $10 \mathrm{~m}$ raster cells. While some of the clear water areas (A03, A04, P07, P12) still show a moderate underestimation (e.g. P12: $-0.04 \mathrm{~m}$ ), some areas even show water level overestimation using the $99 \%$ quantile estimator. As expected, the amount of underestimation generally increases with decreasing cell sizes. While many of the Topo-Bathymetric test areas (e.g. P05, P06, P14) still feature moderate water level underestimation for the $1 \mathrm{~m}$ resolution (e.g. P14: $-0.02 \mathrm{~m}$ ), the water surface point density is generally too low for the Single Photon LiDAR test areas with a mean error ranging from $-0.11 \mathrm{~m}$ (A01) to $-0.21 \mathrm{~m}$ (A05). Furthermore, the mean deviation values reported in Table 3 once again underline that the green laser echoes show a considerable variation of subsurface penetration for the individual water bodies both for Single Photon and Topo-Bathymetric Multi-Photon LiDAR.

\begin{tabular}{|l|r|r|r|r|}
\hline & $\mathbf{1 . 0} \mathbf{~ m}$ & $\mathbf{2 . 0} \mathbf{~ m}$ & $\mathbf{5 . 0} \mathbf{~ m}$ & $\mathbf{1 0 . 0} \mathbf{~ m}$ \\
\hline A01 & -0.11 & -0.06 & 0.00 & 0.02 \\
\hline A03 & -0.16 & -0.11 & -0.05 & -0.02 \\
\hline A04 & -0.17 & -0.17 & -0.09 & -0.03 \\
\hline A05 & -0.21 & -0.16 & -0.06 & -0.03 \\
\hline P02 & -0.06 & -0.02 & 0.01 & 0.02 \\
\hline P05 & -0.04 & -0.02 & -0.01 & 0.00 \\
\hline P06 & -0.02 & 0.00 & 0.01 & 0.02 \\
\hline P07 & -0.15 & -0.09 & -0.06 & -0.03 \\
\hline P11 & -0.05 & -0.03 & -0.02 & -0.01 \\
\hline P12 & -0.15 & -0.10 & -0.06 & -0.04 \\
\hline P14 & -0.02 & -0.01 & 0.00 & 0.01 \\
\hline
\end{tabular}

Table 3. Mean deviations [m] of different digital water surface models from the reference water level for the $99 \%$ quantile.

\section{CONCLUSIONS AND OUTLOOK}

In this contribution we investigated the feasibility of Single Photon LiDAR for large area water surface mapping. In a case study, data from the Spanish province Navarra captured with a SPL100 sensor was compared to Multi-Photon LiDAR data acquired with a VQ-880-G Topo-Bathymetric laser scanner. Both instruments use green laser light $(\lambda=532 \mathrm{~nm})$ to measure objects on land (bare earth, vegetation, buildings, etc.) and water (surface and bottom). While it is known from literature that water surface mapping based on green laser light suffers from the potential penetration of the laser signal into the topmost layer of the water column (Thomas and Guenther, 1990; Zhao et al., 2017; Mandlburger et al., 2013), the specific research questions were if Single Photon LiDAR (i) is less affected by the well known water-surface-underestimation effect due the high receiver sensitivity and (ii) consequently delivers a higher number of water surface echoes.

The question was addressed experimentally by analyzing the 3D point clouds of selected horizontal water bodies (Single Photon LiDAR: reservoirs; Multi-Photon LiDAR: ground water ponds). In both cases, for each water body the following processing steps were applied: quality control including check of strip fitting precision and point density, estimation of a reference water level, interpolation of digital water surface raster models from all points with a water depth less than $50 \mathrm{~cm}$ in resolutions ranging from 1$10 \mathrm{~m}$ (Mandlburger et al., 2013), and statistically analyzing the height deviations of the water surface models from the reference water level. While independently captured ground truth data 
Single Photon LiDAR
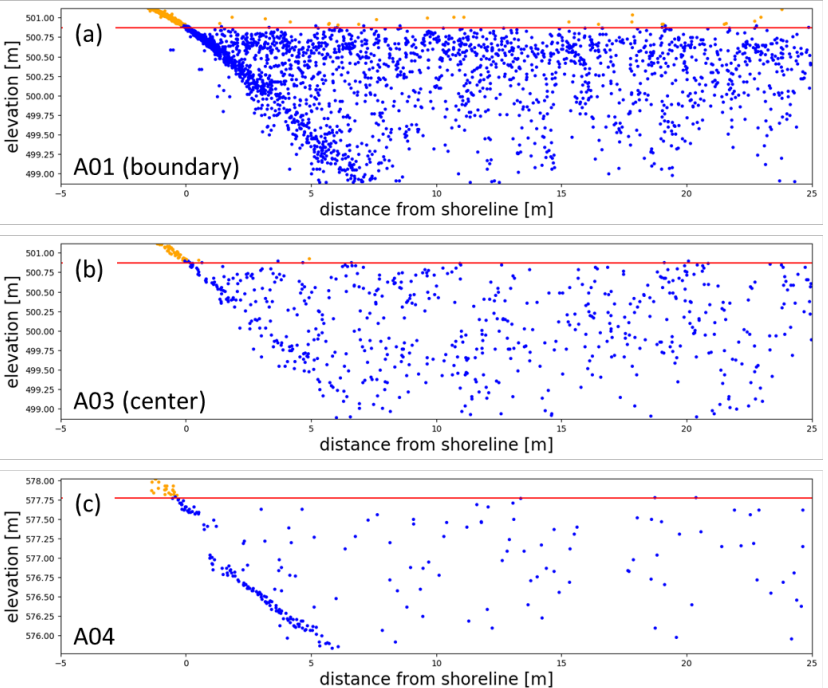

Topo-Bathymetric Multi-Photon LiDAR
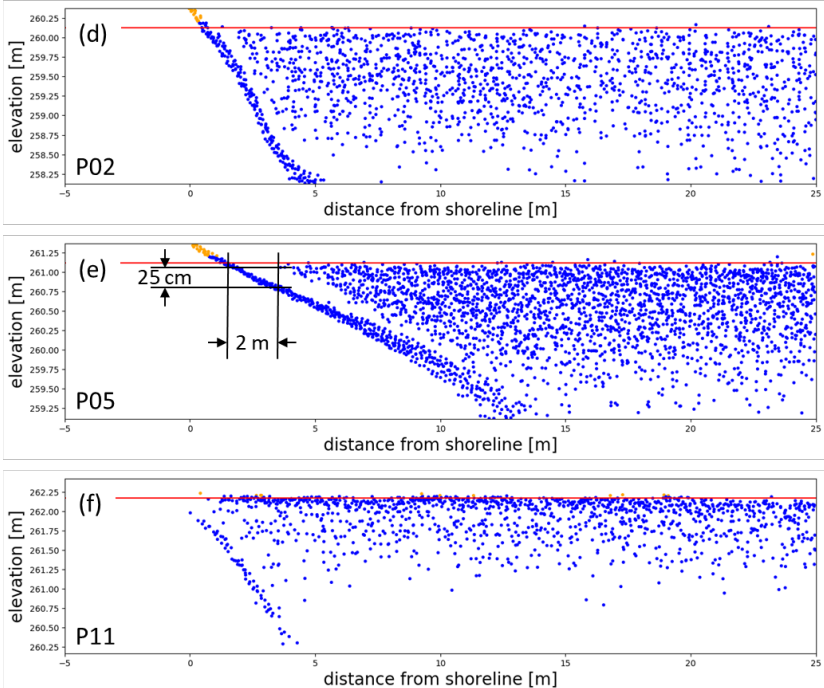

Figure 5. Selected vertical sections; (a-c) Single Photon LiDAR; (d-f) Topo-Bathymetric Multi-Photon LiDAR; blue: water candidate points, orange: points on dry land, red line: estimated water level

q0.90
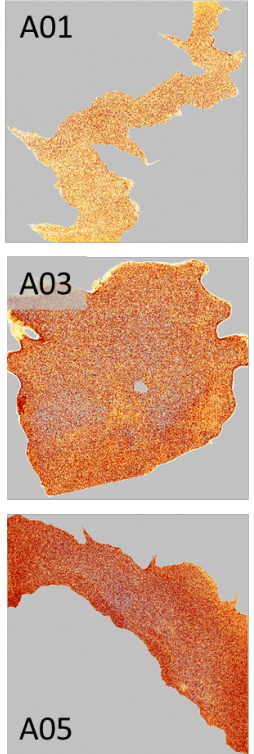

q0.95
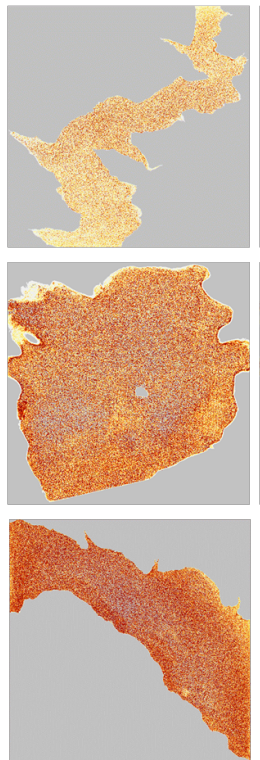

q1.00
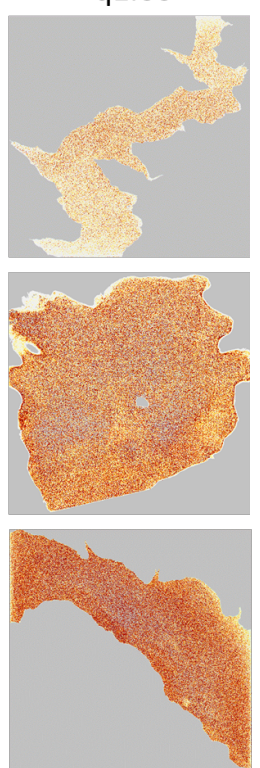

q0.90
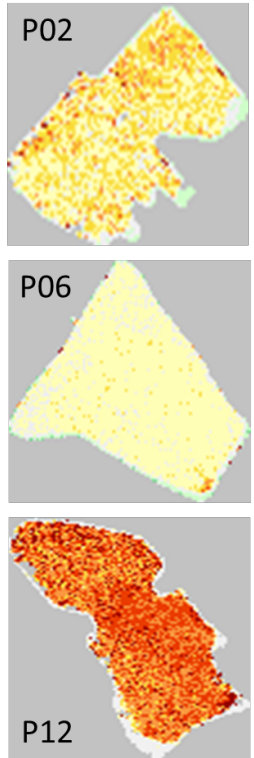

q0.95
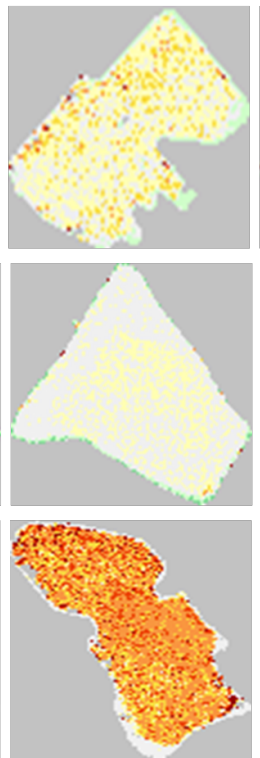

q1.00

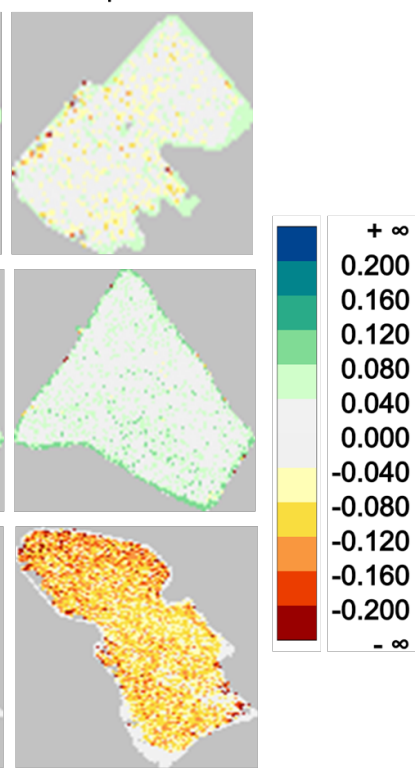

Figure 6. Deviations between reference water level and $2 \mathrm{~m}$ digital water surface model grids calculated for selected Single Photon and Multi-Photon LiDAR water bodies and different water level height quantiles.

would be necessary to reinforce the results, the following conclusions can be drawn from this preliminary investigation:

- Over land, the expected relation w.r.t. local roughness of the point cloud and global flight strip fitting precision between Single Photon LiDAR $(3 \mathrm{~cm})$ and Multi-Photon LiDAR $(1 \mathrm{~cm})$ could be verified. The better precision of MultiPhoton LiDAR stems from the fact that at least 250 photons are necessary for a single measurement while a few photons are sufficient to trigger an echo in the Single Photon case. The higher Multi-Photon LiDAR precision comes at the price of a lower areal coverage performance (single strip swath width: $480 \mathrm{~m}$ vs. $2260 \mathrm{~m}$, scan rate: $550 \mathrm{kHz}$ vs. $6 \mathrm{MHz})$.
- Over water, the last echo point density of Single Photon LiDAR is less compared to Topo-Bathymetric Multi-Photon LiDAR. For Single Photon LiDAR a water point density drop of $50 \%$ compared to the land area was observed, while a respective point density drop for Multi-Photon LiDAR was only observed for very clear water conditions. In both cases a correlation between turbidity and point density was observed, but further experiments are needed to verify the relationship.

- In general, the water level underestimation was less for Multi-Photon LiDAR and the respective (near) water surface point density was less for Single Photon LiDAR.

- For both data sources acceptable agreement between local water surface heights an the reference water level could be achieved when aggregating the near water surface points 
into $10 \times 10 \mathrm{~m}^{2}$ cells and interpolating the surface height within a cell using a high quantile (e.g. $99 \%$ ). Under these preconditions the water surface underestimation was below $4 \mathrm{~cm}$.

- For water surface models with a grid spacing of 1-2 m, Single Photon LiDAR often did not deliver enough near water surface points to obtain a gapless model and Multi-Photon LiDAR outperformed Single Photon LiDAR in terms of precision.

These findings basically mean, that Single Photon LiDAR based large area water level mapping is feasible for surface model resolutions of $5-10 \mathrm{~m}$, but the expected effect that the high receiver sensitivity reduces the underestimation bias compared to MultiPhoton LiDAR could not be verified. However, this statement needs the following clarification. The SPL100 instrument is optimized for capturing topography. The multi-target capabilities of the sensor crucially depend on a well chosen receiver sensitivity level. If chosen too sensitive, most of the sensor array cells will trigger at a very weak level of received signal. However, the low recovery time (1.6ns) reported in the SPL100 data sheet (Leica, 2017) can only be achieved if some photosensitive array elements respond to a (weak) signal of an object while others do not, thus, staying responsive for delayed echos. This having said, a higher receiver sensitivity level might improve the Single Photon based water surface mapping capabilities at the price of a higher level of clutter points from the atmosphere and a poorer topographic point density.

The presented investigation suffered most from the lack of independently measured ground data. Future work on subject matters will therefore concentrate on properly designing experiments with the sole purpose of Single Photon based water surface mapping including the selection of representative water bodies featuring different water surface and water column characteristics (roughness, turbidity, etc.), simultaneous data acquisition of Single and Multi-Photon LiDAR, application specific Single Photon LiDAR flight mission parameter setting (laser energy, FOV, flying height, etc.), and capturing of adequate trustworthy reference data.

\section{ACKNOWLEDGEMENTS}

The presented investigation was conducted within the project "'Bathymetry by Fusion of Airborne Laser Scanning and MultiSpectral Aerial Imagery"' funded by the German Research Foundation (DFG). The authors want to thank Roberto Pascual (Government of Navarra, Cartography Section) and Victor Garcia Morales (Tracasa) for providing the Leica SPL100 data (Navarra) and company RIEGL for providing the Topo-Bathymetric LiDAR data (Pielach/Neubacher $\mathrm{Au})$.

\section{References}

Degnan, J. J., 2016. Scanning, Multibeam, Single Photon Lidars for Rapid, Large Scale, High Resolution, Topographic and Bathymetric Mapping. Remote Sensing 8(11), pp. 958-1-23.

Guenther, G. C., Cunningham, A. G., Laroque, P. E. and Reid, D. J., 2000. Meeting the accuracy challenge in airborne lidar bathymetry. In: Proceedings of the 20th EARSeL Symposium: Workshop on Lidar Remote Sensing of Land and Sea, Dresden, Germany.
Jutzi, B., 2017. Less Photons for More LiDAR? A Review from Multi-Photon Detection to Single Photon Detection. 56th Photogrammetric Week, Stuttgart, Germany, http://www.ifp.unistuttgart.de/phowo/2017/PDF/10-Jutzi-Abstract.pdf.

Jutzi, B. and Stilla, U., 2003. Analysis of laser pulses for gaining surface features of urban objects. In: 2nd GRSS/ISPRS Joint Workshop on Remote Sensing and Data Fusion over Urban Areas. URBAN 2003, Berlin, IEEE, Piscataway, New Jersey, pp. 13-17.

Jutzi, B. and Stilla, U., 2005. Waveform processing of laser pulses for reconstruction of surfaces in urban areas. In: $I S$ PRS Joint Conference 3rd International Symposium Remote Sensing and Data Fusion over Urban Areas (URBAN 2005), 5th International Symposium Remote Sensing of Urban Areas (URS 2005), Tempe, Arizona, USA, 6 pp.

Leica, 2017. SPL100 single photon LiDAR sensor data sheet. Leica Geosystems, Heerbrugg, Switzerland. https://leica-geosystems.com/products/airborne-systems/lidarsensors/leica-spl100 (last accessed: 2018-07-14).

Mandlburger, G., Hauer, C., Wieser, M. and Pfeifer, N., 2015. Topo-Bathymetric LiDAR for Monitoring River Morphodynamics and Instream Habitats-A Case Study at the Pielach River. Remote Sensing 7(5), pp. 6160-6195.

Mandlburger, G., Pfennigbauer, M. and Pfeifer, N., 2013. Analyzing near water surface penetration in laser bathymetry A case study at the River Pielach. In: ISPRS Annals of the Photogrammetry, Remote Sensing and Spatial Information Sciences, Vol. II-5/W2, pp. 175-180.

Pfeifer, N., Mandlburger, G., Otepka, J. and Karel, W., 2014. OPALS - A framework for Airborne Laser Scanning data analysis. Computers, Environment and Urban Systems 45, pp. 125 $-136$.

Pfennigbauer, M. and Ullrich, A., 2010. Improving quality of laser scanning data acquisition through calibrated amplitude and pulse deviation measurement. In: Proc. SPIE, Vol. 7684, pp. $7684-7684-10$.

Riegl, 2017. VQ-880-G topo-bathymetric LiDAR sensor data sheet. Riegl Laser Measurement Systems, Horn, Austria. http://www.riegl.com/nc/products/airbornescanning/produktdetail/product/scanner/46/ (last accessed: 2018-07-14).

Rupnik, E., Jansa, J. and Pfeifer, N., 2015. Sinusoidal wave estimation using photogrammetry and short video sequences. Sensors 15(12), pp. 30784-30809.

Stoker, J. M., Abdullah, Q. A., Nayegandhi, A. and Winehouse, J., 2016. Evaluation of Single Photon and Geiger Mode Lidar for the 3D Elevation Program. Remote Sensing 8(9), pp. 767$1-16$.

Thomas, R. W. L. and Guenther, G. C., 1990. Water surface detection strategy for an airborne laser bathymeter. In: Proc. SPIE, Vol. 1302, pp. 1302-1-15.

Tulldahl, H. M. and Steinvall, K. O., 2004. Simulation of sea surface wave influence on small target detection with airborne laser depth sounding. Appl. Opt. 43(12), pp. 2462-2483.

Ullrich, A. and Pfennigbauer, M., 2016. Linear LIDAR versus Geiger-mode LIDAR: impact on data properties and data quality. In: Proc. SPIE, Vol. 9832, pp. 983204-1-17.

Zhao, J., Zhao, X., Zhang, H. and Zhou, F., 2017. Shallow water measurements using a single green laser corrected by building a near water surface penetration model. Remote Sensing 9(5), pp. 426-443. 